\title{
The Effects of Clonidine on Discrete-Trial Delayed Spatial Alternation in Two Rat Models of Memory Loss
}

\author{
Mark E Bardgett*,', Megan Points', Christian Ramsey-Faulkner', Jeff Topmiller', John Roflow', \\ Travis McDaniel', Timberly Lamontagne' and Molly S Griffith' \\ 'Department of Psychology, Northern Kentucky University, Highland Heights, KY, USA
}

\begin{abstract}
Spatial memory impairments observed in Alzheimer's disease and schizophrenia have been attributed to many factors, including glutamate hypofunction and reduced hippocampal volume. Clonidine, a non-specific $\alpha_{2}$ adrenergic receptor agonist, improves spatial memory in animals treated with the N-methyl-D-aspartate (NMDA) receptor antagonist, phencyclidine; however, its effects on memory deficits produced by other NMDA antagonists or hippocampal damage have not been fully characterized. The purpose of this study was to determine if clonidine could alleviate memory deficits produced by the NMDA antagonist, MK-80 I, or by excitotoxic hippocampal damage. In the first phase of the study, male rats were pretreated with clonidine $(0.01$ or $0.05 \mathrm{mg} / \mathrm{kg})$ or saline, and treated with $\mathrm{MK}-80 \mathrm{I}$ $(0.1 \mathrm{mg} / \mathrm{kg})$ or saline prior to discrete-trial delayed alternation or radial-arm maze testing. MK-80I impaired delayed alternation performance and increased the number of arm revisits in the radial-arm maze. Clonidine pretreatment significantly alleviated these druginduced deficits. In the second phase of the study, excitotoxic damage was produced in the dorsal hippocampus with NMDA. Hippocampal damage produced a significant impairment in the delayed alternation task, yet pretreatment with clonidine did not alleviate this damage-induced deficit. Taken together, the data indicate that clonidine alleviates memory impairments produced by glutamate hypofunction, but not by hippocampal damage. This caveat may be important in designing treatments for memory disorders not linked to a single pathophysiological mechanism.

Neuropsychopharmacology (2008) 33, 1980-199I; doi:I0.1038/sj.npp. I30I580; published online 19 September 2007
\end{abstract}

Keywords: NMDA; hippocampus; spatial memory; norepinephrine; schizophrenia; Alzheimer's disease

\section{INTRODUCTION}

In a seminal article, Hyman and Fenton (2003) argued that the recognition and treatment of cognitive impairment should be placed at the forefront of research into neuropsychiatric disorders. This argument was founded upon data showing that cognitive impairment in neuropsychiatric disorders strongly predicts functional outcome (Hyman and Fenton, 2003). To address this call for better treatment for cognitive impairment, it is incumbent to develop new animal models of cognitive impairment as well as to assess candidate compounds in a variety of extant memory loss models. By using multiple models, one can better understand the kinds of pathophysiology that can be corrected by such drugs, and as importantly, the types of pathophysiological processes that are resistant to them. The research reported here was directed at this goal-the

*Correspondence: Dr ME Bardgett, Department of Psychology, Northern Kentucky University, I Nunn Drive, Highland Heights, KY 4l076, USA, Tel: + I 859572 559|, Fax: + | 859572 6085,

E-mail: bardgettm@nku.edu

Received 30 April 2007; revised 14 August 2007; accepted I5 August 2007 assessment of clonidine, a potential cognitive-enhancing drug, in two animal models of memory dysfunction.

Glutamate hypofunction in animals has been used as a model of memory impairment over the past 20 years. This model is based on the hypothesis that reduced activity at $\mathrm{N}$-methyl-D-aspartate (NMDA) receptors accounts for the cognitive deficits found in schizophrenia and Alzheimer's disease (Lewis and Moghaddam, 2006; Olney and Farber, 1995; Olney et al, 1998). This hypothesis is supported by clinical work demonstrating that NMDA receptor antagonists, such as ketamine, can decrease declarative memory performance (Newcomer et al, 1999). In animals, phencyclidine (PCP) or MK-801 is typically used to block NMDA receptors, and these drugs significantly impair performance in delayed spatial alternation (Aultman and Moghaddam, 2001; Moghaddam and Adams, 1998) and radial-arm maze tasks (Zhang et al, 2005). The effects of these antagonists on spatial working memory may be mediated through specific brain regions, such as the medial prefrontal cortex and hippocampus, since microinfusions of NMDA antagonists into either region can disrupt this form of memory (Aura and Riekkinen, 1999; Yoshihara and Ichitani, 2004). To date, the NMDA hypofunction model has been useful in identifying potential memory-enhancing drugs with novel 
mechanisms of action, such as acetylcholinesterase inhibitors (Csernansky et al, 2005; Keseberg and Schmidt, 1993), agonists at group II metabotropic glutamate receptors (Moghaddam and Adams, 1998), histamine $\mathrm{H}_{3}$ antagonists (Bernaerts et al, 2004), combinations of $\alpha_{2}$ adrenergic/ $\mathrm{D}_{2}$ dopamine receptor antagonists (Marcus et al, 2005), and agonists at $\alpha_{2}$ adrenergic receptors (Jentsch and Anzivino, 2004; Marrs et al, 2005; McCann et al, 1987).

A complimentary approach to a model of memory impairment based on neurochemical dysfunction is a model based on localized neuroanatomical changes. One of the most consistently localized neuroanatomical changes found in neuropsychiatric disorders is reduced hippocampal volume, although it should be emphasized that structural decrements have been observed in other brain regions, including the prefrontal cortex and other areas of the temporal lobe (McCarley et al, 1999). Studies of people with schizophrenia, Alzheimer's disease, and depression have shown that the size of the hippocampus is reduced in comparison to individuals without these disorders (Csernansky et al, 2000; McCarley et al, 1999; Sheline et al, 1996). Moreover, correlative neuroimaging studies have revealed associations between reduced hippocampal volume and poorer cognitive performance in schizophrenia (Gur et al, 2000; Heckers, 2001) and Alzheimer's disease (Grundman et al, 2002; Kohler et al, 1998). Given this association, it would seem worthwhile to use animals with experimental hippocampal damage (Bardgett et al, 2006a,b) to identify treatments for memory problems related to reduced hippocampal volume.

In terms of choosing drugs to assay in the NMDA hypofunction and hippocampal damage models, agonists at $\alpha_{2}$ adrenergic receptors are among the most promising candidates. Clinical studies have shown that the $\alpha_{2}$ agonist, clonidine, can improve some aspects of working memory in people with Alzheimer's disease (Riekkinen and Riekkinen, 1999; although see Riekkinen et al, 1999) and schizophrenia (Friedman et al, 2001). A related $\alpha_{2}$ agonist, guanabenz, prevents working memory deficits in normal people given the NMDA antagonist, ketamine (Newcomer et al, 1999). While it has been shown in rats that $\alpha_{2}$ agonists such as clonidine and guanfacine attenuate spatial working memory impairments caused by PCP (Jentsch and Anzivino, 2004; Marrs et al, 2005; McCann et al, 1987), it is unknown whether $\alpha_{2}$ agonists can reverse the deleterious effects of other NMDA antagonists, such as MK-801, on memory. Moreover, there is little information on the ability of $\alpha_{2}$ agonists to improve memory in animals with hippocampal damage.

Given the beneficial effects of clonidine and related $\alpha_{2}$ agonists on prefrontal cortical function (Li et al, 1999; Wang et al, 2007) as well as the important role of the prefrontal cortex in the positive actions of $\alpha_{2}$ agonists on spatial working memory (Arnsten and Goldman-Rakic, 1985; see Arnsten, 2004 for review), it is likely that if clonidine improves memory in either model, this phenomenon would be linked to enhanced prefrontal cortical function. In the case of animals with hippocampal damage, the enhancement of prefrontal function could act to compensate for the loss of hippocampal modulation of spatial working memory. This idea receives indirect support from two lines of research. First, hippocampal damage appears to render the prefrontal cortex more sensitive to specific types of stimulation. Rats with kainic acid-induced hippocampal damage demonstrate an increased sensitivity to antipsychotic drug-induced transcriptional activity in the prefrontal cortex (Roe et al, 1998) while rats with neonatal ventral hippocampal lesions are more sensitive to dopaminergic input in the same region (O'Donnell et al, 2002). Second, work, as reviewed by Kesner and Rogers (2004), has shown that the medial prefrontal cortex can compensate for short-term working memory loss induced by transient inactivation of the hippocampus. In cases of more severe hippocampal damage wherein spatial memory impairment is permanent, it appears that the prefrontal cortex is not sufficient to correct this deficiency. But the augmentation of neurotransmission in the prefrontal cortex provided by drugs, such as clonidine, could conceivably enable this brain region to adequately process working memory demands normally handled by hippocampal neurons, leading to improved memory in animals with hippocampal damage.

The purpose of this study was to determine if clonidine could attenuate the spatial working memory deficits produced by the NMDA antagonist, MK-801, or by direct excitotoxic damage to the dorsal hippocampus. To achieve this goal, the effects of clonidine on performance in a discrete-trial, delayed spatial alternation task were measured in rats after acute treatment with MK-801 or in a separate cohort of rats with and without hippocampal damage. Furthermore, the effects of clonidine on performance in an eight-arm radial maze were determined in rats after MK-801 treatment. The discrete-trial delayed alternation task and the radial-arm maze were chosen since numerous studies have demonstrated decrements in memory after hippocampal damage or NMDA receptor antagonist administration (Adams and Moghaddam, 1998; Dudchenko, 2001; Kretschmer and Fink, 1999; Moghaddam et al, 1997). In the present studies, the doses of clonidine and MK-801, and the timing of drug administration were similar to the doses and drug administration schedules used in previous studies of spatial memory involving either drug (Ammassari-Teule et al, 1991; Bardgett et al, 2003; Jentsch and Anzivino, 2004).

\section{MATERIALS AND METHODS}

\section{Animals}

Young adult male Long-Evans rats, $60-75$ days of age at the beginning of the study, were purchased from Harlan (Harlan Bioproducts Inc, Indianapolis, IN). They were housed three per cage with free access to food and water except during testing. Lighting was maintained on a 12-h schedule with lights on at 0700 hours. Experimental procedures were performed according to the Guide for the Care and Use of Laboratory Animals (National Research Council, 1996) under a protocol approved by the Northern Kentucky University Institutional Animal Care and Use Committee.

\section{Discrete-Trials Rewarded Delayed Spatial Alternation}

The T-maze used in these experiments was constructed of wood and painted black. It contained $10 \mathrm{~cm}$ high walls and 
$9 \mathrm{~cm}$ wide corridors. The stem was $40 \mathrm{~cm}$ long, each arm was $45 \mathrm{~cm}$ long, and the entire maze had a wire mesh bottom. A week prior to habituation and testing, all animals were placed on a food-restricted diet and reduced to $\sim 90 \%$ of their free-feeding weight. During habituation, all animals were placed on the T-maze until they ate two pieces of food or $90 \mathrm{~s}$ had elapsed. This was repeated three times a day with a 5-min intertrial interval. During testing, animals received six trials a day for 4 days a week. There was a 5min intertrial interval. Each test trial consisted of two runs, a forced run and a free run. On the forced run, rats were forced to obtain a piece of food from one goal arm of the Tmaze. Goal arm entries were defined as placing four paws in the arm. A total of 10 or $40 \mathrm{~s}$ after the end of the forced run, the free run was conducted. At the beginning of the free run, the rat was returned to the start arm and allowed to choose either goal arm. If the rats chose the arm opposite of the one they had been forced into during the forced run, they received the food reward. If the rats chose the same arm that they had been forced into, they received no food reward. Rats were given three 10 -s and three 40 -s delay trials during each day of testing. The sequence of delays and forced run food locations (left or right) were randomized each day, with the stipulation that the same delay or the same forced arm location could not be used for three trials in a row. All animals were tested for at least 1 week before drug testing began. The time to choose a goal arm was measured as a gross assessment of potential drug effects on sensorimotor function. On each run, the time from the placement of the rat on the maze until the rat placed four paws into one of the goal arms was recorded. The average time to choose across all runs on a given test day was then determined for each animal.

\section{Radial-Arm Maze Testing}

An eight-arm maze was constructed of clear Plexiglas and wood that was painted black. Each arm was $120 \mathrm{~cm}$ high, $120 \mathrm{~cm}$ long, and $10 \mathrm{~cm}$ wide. The center platform of the maze was $30 \mathrm{~cm}$ in diameter. The surface of the arms and center platform was Plexiglas. Plexiglas doors could be placed at the beginning of each arm near the center platform in order to prohibit entry into a given arm. All animals were placed on a food-restricted diet 1 week before habituation to the maze began. One habituation trial was performed for 5 consecutive days. Before each trial, 1/2 of a Cheerio was placed in the middle of each arm of the maze and another was placed at the end of each arm. The rat was removed from the maze after eating all of the food or after $10 \mathrm{~min}$ had elapsed. For testing, a delayed non-matching-tosample procedure based on the work of Liu and Bilkey (1999) was used. Each trial consisted of a forced run and a free choice run. On the forced run, four arms of the maze were baited with $1 / 2$ of a Cheerio and the other four arms were blocked with the Plexiglas doors. The sequence of open and blocked arms was randomly selected each day of testing. Each rat was placed in the center platform and given $5 \mathrm{~min}$ to eat the four pieces of food. The number of revisits to arms previously visited was recorded. After a delay of $30 \mathrm{~s}$ or $15 \mathrm{~min}$, the animal was returned to the center platform and allowed access to all eight arms. Food rewards were placed only in the arms that were previously blocked off.
The animal was given $5 \mathrm{~min}$ to eat the four pieces of food. The following data were recorded during the second run: (1) the number of correct responses during the first four arm choices, (2) the number of retroactive errors (ie visits to arms that were baited during the forced run), and (3) the number of revisits to arms that were baited during the free choice run. Two trials (one $30 \mathrm{~s}$ and one $15 \mathrm{~min}$ delay) were run per day with a 30-min intertrial interval. Animals were tested for 10 days before the start of drug treatments.

\section{NMDA-Induced Hippocampal Damage and Histology}

Stereotaxic surgery was performed in order to infuse NMDA (Sigma, St Louis, MO, USA) into the dorsal hippocampus of the rats. NMDA was dissolved daily into $0.9 \%$ sterile saline. Each rat was placed in a Kopf stereotaxic frame following the administration of Nembutal (Abbott, North Chicago, IL) $(75 \mathrm{mg} / \mathrm{kg}, \mathrm{IP})$. NMDA $(3.0 \mathrm{mg} / \mathrm{ml})$ or vehicle was injected at four bilateral locations (AP: $-2.3,-3.4,-4.3,-5.2 ; \mathrm{ML}$ : $\pm 1.9, \pm 2.2, \pm 3.3, \pm 3.9 ; \mathrm{V}:-3.5,-3.6,-3.9,-4.1)$ along the longitudinal axis of the hippocampal formation. A total of $0.3 \mu \mathrm{l}$ of NMDA solution or vehicle was injected into each location over $2 \mathrm{~min}$ and the needle remained in place for an additional minute. Animals were allowed to recover for 1 month before the beginning of the delayed alternation task. The presence and extent of hippocampal damage was verified and quantified in each animal using histological methods previously described (Bardgett et al, 2006a, b).

\section{Experiment 1: The Effects of MK-801 on Choice Accuracy in the Delayed Alternation Task}

This experiment was performed to determine the effects of two doses of MK-801 (0.05 and $0.1 \mathrm{mg} / \mathrm{kg})$ vs saline on choice accuracy in the delayed alternation task. A total of 15 drug-naïve rats were used in this experiment. MK-801 (Sigma) was dissolved in $0.9 \%$ saline, and injected subcutaneously into animals $30 \mathrm{~min}$ prior to testing. Animals received the three drug treatments in a counterbalanced order across testing days. Each animal was tested once in each drug condition over a 3-day period.

\section{Experiment 2: The Effects of Clonidine on MK-801-Induced Deficits in Delayed Alternation Task}

A total of 12 rats from the first experiment were used in the second experiment. Based on the results of the first experiment, a dose of $0.1 \mathrm{mg} / \mathrm{kg}$ of $\mathrm{MK}-801$ was chosen for further study. A total of 3 days after completing the first experiment, the second experiment was conducted over 6 days with a 3-day break between the third and fourth day of testing. Clonidine (Sigma) was dissolved in saline and injected subcutaneously $30 \mathrm{~min}$ before $\mathrm{MK}-801$ or saline injection. Testing began $30 \mathrm{~min}$ after the latter injection. Doses of clonidine were chosen $(0.01$ and $0.05 \mathrm{mg} / \mathrm{kg})$ that have been found to effectively improve memory in other animal models of memory loss (Ammassari-Teule et al, 1991; Jentsch and Anzivino, 2004). The order of pretreatment and treatment was counterbalanced across days, and each animal was tested once under each of the four possible pretreatment/treatment combinations. 
Experiment 3: The Effects of Clonidine on MK-801-Induced Deficits in the Radial-Arm Maze

A total of six drug-naïve rats were used in this experiment. After 10 days of testing without drug treatment, animals received subcutaneous injections of clonidine $(0.05 \mathrm{mg} / \mathrm{kg})$ or saline $60 \mathrm{~min}$ before testing and subcutaneous injections of MK-801 $(0.1 \mathrm{mg} / \mathrm{kg})$ or saline $30 \mathrm{~min}$ before testing began. Animals were tested over a period of 16 days and received two trials each day after pretreatment/treatment injections. The order of pretreatment and treatment was counterbalanced across days, and each animal was tested four times under each of the four possible pretreatment/ treatment combinations.

Experiment 4: The Effects of Clonidine on Hippocampal Damage-Induced Deficits in the Delayed Alternation Task

There were 29 drug-naïve rats used in this experiment with 13 rats in the hippocampal damage group and 16 rats in the sham control group. At 5 weeks post-surgery, after animals were acclimated to the same food-restriction and habituation procedures described above, the animals were tested for 2 weeks without any drug treatment. Animals were then tested four times a week for 3 weeks. During each week of testing, animals received a different dose of clonidine ( 0.01 or $0.05 \mathrm{mg} / \mathrm{kg}$ ) or saline for 4 consecutive days (ie the animal received the same drug dose for 4 days in a row and was then switched to a different drug dose during the following week). The order of drug treatment was counterbalanced within groups across weeks. Animals received injections $30 \mathrm{~min}$ prior to testing.

\section{Data Analyses}

In the experiments examining the effects of clonidine and MK-801 in the delayed alternation task and the radial-arm maze, a three-way, repeated measures ANOVA was used. All main factors were treated as within-group factors, including pretreatment (different doses of clonidine and saline), treatment (MK-801 vs saline), and delay interval. In the first and second experiments, the time to choose a goal arm was compared between treatment groups and pretreatment groups (second experiment only) with repeated measures analysis of variance (ANOVAs). In the experiment examining the effects of clonidine on delayed alternation performance in rats with hippocampal damage, a two-way ANOVA was performed with treatment serving as a within-groups factor and hippocampal or sham damage serving as a between-groups factor. Relative hippocampal area was compared between animals with and without hippocampal damage as a function of anterior-posterior location with two-way repeated measures ANOVA, with damage as a between-groups factor and section location as a within-groups factor. All post hoc comparisons were conducted when appropriate by using one-way ANOVA's and Fishers Protected Least Significant Difference (PLSD) test. $\alpha$-levels were set at $p<0.05$ (two-tailed) for all tests.

\section{RESULTS}

MK-801 Impairs Choice Accuracy in the Discrete-Trial Delayed Alternation Task

In the first set of experiments, MK-801 was found to significantly impair choice accuracy in the discrete-trial delayed alternation task in a dose-dependent manner (Figure 1). There was an overall effect of MK-801 on choice accuracy (drug effect: $\mathrm{F}(2,68)=6.8, p<0.002$ ) as well as a significantly effect of delay (delay effect: $F(1,34)=15.0$, $p<0.0005$ ). At the 10 -s delay, there were no significant differences in choice accuracy between the drug-treated groups. However, at the 40 -s delay, the animals treated with the high dose of MK-801 performed significantly worse than animals treated with saline or the low dose of MK-801 (Fishers PLSD, $p<0.0007$ and 0.002 in respective comparisons to the high dose MK-801 group). Within drug treatment groups, there was no significant delay effect on animals treated with saline, but animals treated with the low and high dose of MK-801 made significantly fewer correct choices at the 40-s delay than at the 10-s delay (Fishers PLSD, $p<0.02$ and 0.006 for the respective comparisons across delays within the low and high dose MK-801 groups). While MK-801 impaired choice accuracy, it was not found to significantly alter the time to choose a goal arm (Table 1).

\section{Clonidine Alleviates MK-801-Induced Impairment in the Discrete-Trial Delayed Alternation Task}

The $0.1 \mathrm{mg} / \mathrm{kg}$ dose of MK-801 was chosen for further study in subsequent experiments given its robust effect on choice accuracy. Two analyses were conducted to ascertain the effects of previous testing alone and previous exposure to MK-801 on delayed alternation performance in this experiment. First, animals pretreated with saline and treated with

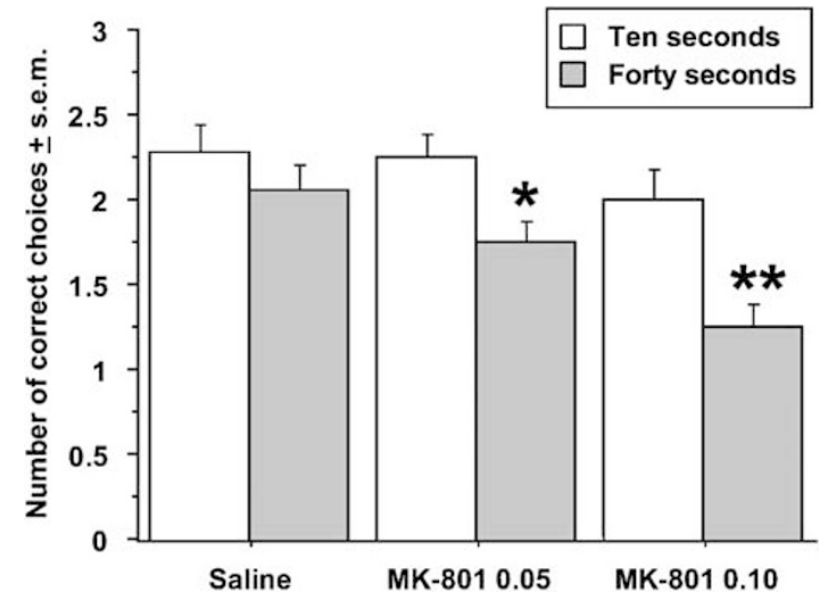

Figure I Effects of MK-80I treatment on choice accuracy in the discrete-trial delayed spatial alternation task. Animals were tested at two delay intervals: 10 and $40 \mathrm{~s}$. The single asterisk (*) indicates that animals treated with the low dose $(0.05 \mathrm{mg} / \mathrm{kg})$ of MK- 80 I made significantly fewer correct choices after the 40-s delay relative to the 10-s delay. The double asterisks (**) indicate that animals treated with the high dose $(0.1 \mathrm{mg} / \mathrm{kg})$ of MK-80I made significantly fewer correct choices after the 40-s delay relative to the 10-s delay and relative to saline-treated rats after the 40-s delay. Data represent the average number of correct choices $( \pm$ SEM) for each group at each delay out of three trials; $n=15$ per group. 
Table I Effects of MK-80 I on the Time to Choose a Goal Arm in the Discrete-Trial Delayed Alternation Task

\begin{tabular}{lc}
\hline Group & Time to choose \\
\hline Saline & $3.0 \pm 0.3$ \\
MK-80I $0.05 \mathrm{mg} / \mathrm{kg}$ & $2.5 \pm 0.3$ \\
MK-80I $0.1 \mathrm{mg} / \mathrm{kg}$ & $3.2 \pm 0.2$ \\
\hline
\end{tabular}

$n=15$ per group. The data represent time in seconds \pm SEM. There were no significant differences between the groups.

saline in the second experiment made significantly more correct choices at both delays than animals treated with saline only in the first experiment (experiment effect: $\mathrm{F}(1,20)=4.5, p<0.05)$ (data not shown), suggestive of a practice effect across experiments. Second, animals pretreated with saline and treated with $0.1 \mathrm{mg} / \mathrm{kg}$ of MK-801 in the second experiment made the same number of correct choices at both delays as did animals treated with the same dose of MK-801 in the first experiment (experiment effect: $\mathrm{F}(1,20)=0.3, p=0.58$ ) (data not shown). This finding would seem to indicate that treatment with MK-801 in the first experiment did not alter responses to the drug in the second experiment.

The second experiment determined if clonidine could counteract the detrimental effect of MK-801 on choice accuracy in the delayed spatial alternation task. There were significant effects of clonidine pretreatment and MK-801 treatment at the 10-s delay and a trend toward a pretreatment by treatment interaction (pretreatment effect: $\mathrm{F}(2,44)=3.6, \quad p<0.04$; treatment effect: $\mathrm{F}(1,22)=8.0$, $p<0.01$; interaction effect: $\mathrm{F}(2,44)=2.6, \quad p=0.08)$ (Figure 2a). Animals pretreated with saline and treated with MK-801 made significantly fewer correct choices in comparison to animals in all other experimental groups (Fishers PLSD, $p<0.01-0.0001$ ). There were no other group differences in the 10-s delay experiment. At the 40-s delay, there were significant effects of clonidine pretreatment and MK-801 treatment as well as a significant pretreatment by treatment interaction (pretreatment effect: $\mathrm{F}(2,44)=4.1$, $p<0.001$; treatment effect: $\mathrm{F}(1,22)=24.2, p<0.0001$; interaction effect: $\mathrm{F}(2,44)=5.4, p=0.008$ ) (Figure 2b). As observed after the 10-s delay, animals pretreated with saline and treated with MK-801 made significantly fewer correct choices than animals in all of the other experimental groups (Fishers PLSD, $p<0.01-0.001$ ). Unlike the 10-s delay experiment, animals in the clonidine pretreatment groups that were subsequently treated with MK-801 performed significantly worse than animals pretreated and treated with saline (Fisher PLSD, $p<0.03$ and 0.05 for the low and high dose clonidine/MK-801 groups compared, respectively, to the saline/saline group). The clonidine/MK-801 groups also performed worse in comparison to the animals pretreated with the low dose of clonidine and treated with saline (Fisher PLSD, $p<0.05$ and 0.01 for the low and high dose clonidine/MK-801 groups relative to the low dose clonidine/ saline group).

The time to choose a goal arm was not significantly altered by clonidine or MK-801 alone (Table 2). However, there was a significant pretreatment $\times$ treatment interaction
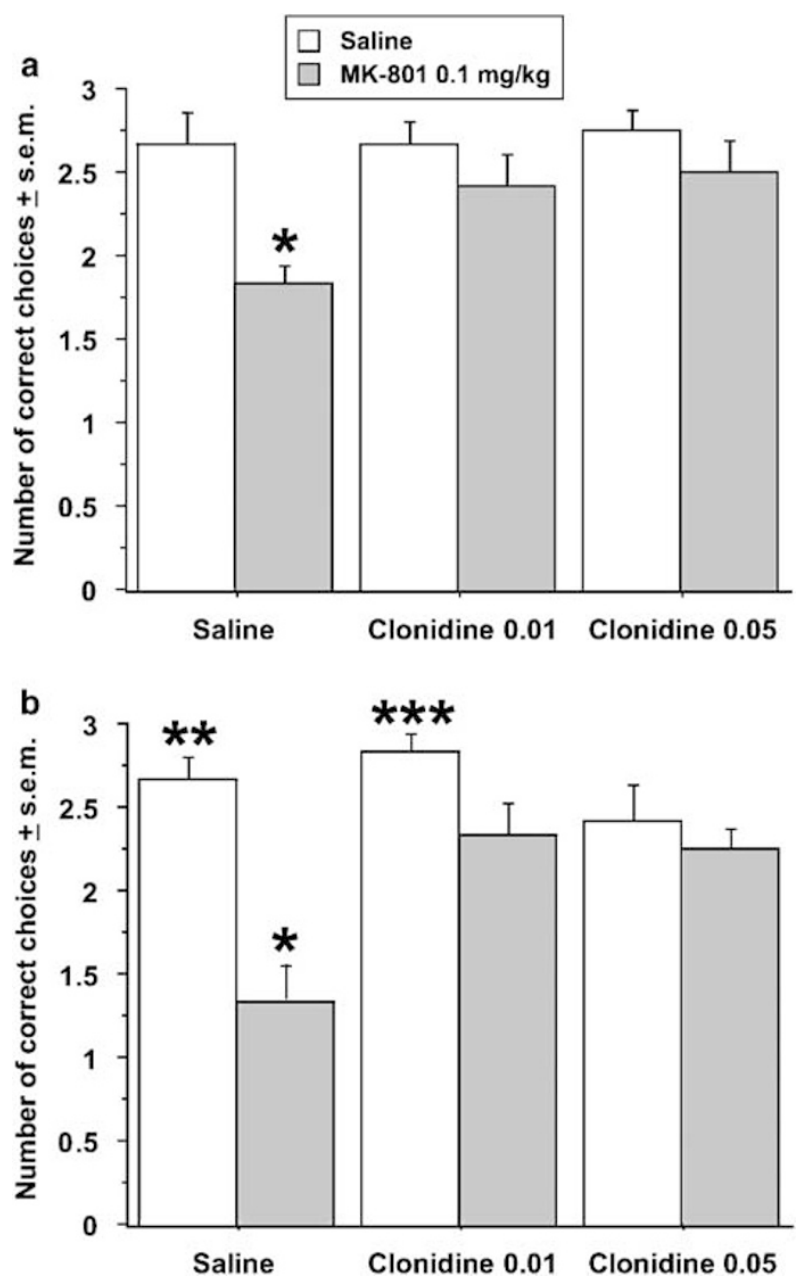

Figure 2 Effects of clonidine pretreatment on deficits produced by MK801 treatment in the discrete-trial delayed spatial alternation task. Data from the 10-s delay are represented in (a) and data from the 40-s delay are represented in (b). The single asterisk (*) in each graph indicates that animals in the saline/MK-80 I group had significantly lower scores relative to all other experimental groups. The double asterisks $(* *)$ in (b) indicate that animals in the saline/saline group had significantly higher scores than animals in each clonidine pretreatment group treated with MK-80I. The triple asterisks (***) in (b) indicate that the latter two groups were significantly lower than animals pretreated with clonidine $0.01 \mathrm{mg} / \mathrm{kg}$ and treated with saline. Data represent the average number of correct choices $( \pm$ SEM) for each group at each delay out of three trials; $n=12$ per group.

(interaction effect: $\mathrm{F}(2,44)=3.7, p<0.03)$. MK-801-treated rats that were pretreated with the low dose of clonidine were significantly quicker in choosing a goal arm than MK-801treated rats pretreated with the high dose of clonidine (Fishers PLSD, $p<0.02$ ). Moreover, saline-treated rats pretreated with the high dose of clonidine were slower to choose an arm relative to saline-treated rats pretreated with saline (Fishers PLSD, $p<0.03$ ).

\section{The Effects of Clonidine and MK-801 on Choice Accuracy in the Radial-Arm Maze}

Clonidine attenuated one of the behavioral deficits produced by MK- 801 in the radial-arm maze. During the first run on each trial, rats were allowed to enter only four arms to obtain food. MK-801 caused rats to make significantly 
more arm revisits during these runs (treatment effect: $\mathrm{F}(1,10)=14.1, p<0.004$ ) (Figure 3a). However, pretreatment with clonidine significantly reduced the number of revisits observed in MK-801-treated rats (pretreatment $x$ treatment interaction: $\mathrm{F}(1,10)=11.2, p<0.007)$. Rats pretreated with clonidine and treated with MK-801 made

Table 2 Effects of Clonidine Pretreatment and MK-80।

Treatment on the Time to Choose a Goal Arm in the DiscreteTrial Delayed Alternation Task

\begin{tabular}{lll}
\hline & \multicolumn{2}{c}{ Treatment group } \\
\cline { 2 - 3 } & Saline & MK-80 I \\
\hline Pretreatment group & & \\
Saline & $4.7 \pm 0.9 *$ & $6.7 \pm 1.3$ \\
Clonidine $0.01 \mathrm{mg} / \mathrm{kg}$ & $6.0 \pm 0.9$ & $3.8 \pm 0.4 * *$ \\
Clonidine $0.05 \mathrm{mg} / \mathrm{kg}$ & $7.1 \pm 0.9$ & $6.2 \pm 0.8$ \\
\hline
\end{tabular}

$n=12$ per group. The data represent the time to choose a goal arm in seconds $( \pm$ SEM). The single asterisk $(*)$ indicates a significant difference between the saline/saline and the clonidine 0.05 /saline groups. The double asterisks (***) indicate a significant difference between the clonidine $0.01 / \mathrm{MK}-80 \mathrm{I}$ and the clonidine 0.05/MK-80 I groups. significantly fewer revisits relative to rats pretreated with saline and treated with MK-801 (Fishers PLSD, $p<0.03$ ).

A total of $30 \mathrm{~s}$ or $15 \mathrm{~min}$ after the first run in each trial, animals were allowed to enter all eight arms, but only the four previously inaccessible arms were baited. While significant delay and treatment effects were identified for the three measures taken during the second run, there were no effects of clonidine or pretreatment $\times$ treatment interactions. There was a delay effect on the number of baited arms chosen (correct choices) within the first four choices (Figure 3b). Rats chose significantly more baited arms among their first four choices after the 30-s delay in contrast to the 15-min delay (Delay effect: $\mathrm{F}(1,20)=15.5$, $p<0.0008)$. Rats were also more likely to visit arms that had been baited during the first run (retroactive error) after the longer delay (Delay effect: $\mathrm{F}(1,20)=4.5, p<0.05$ ) or if the animal was treated with MK-801 (treatment effect: $\mathrm{F}(1,20)=8.9, p<0.007$ ) (Figure 3c). However, for each pretreatment at a given delay interval, individual comparisons between animals treated with saline and those treated with MK-801 were not significant. Finally, MK-801 increased the likelihood that, independent of delay interval, rats would revisit one of the four arms that were baited during the second run (treatment effect: $F(1,20)=11.1$, $p<0.003$ ) (Figure 3d). Individual comparisons of revisits indicated that among saline-pretreated animals at the
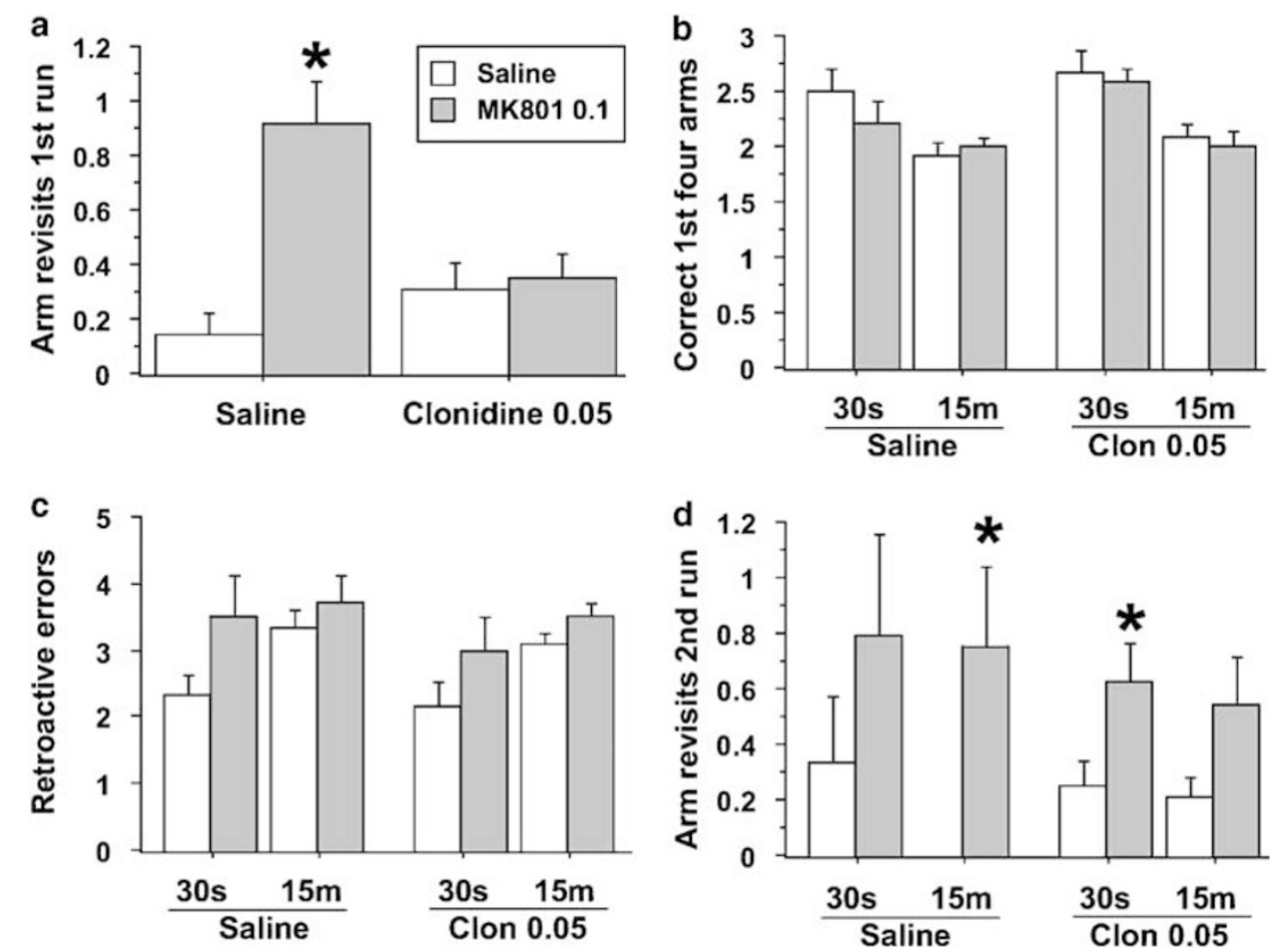

Figure 3 Effects of clonidine pretreatment and MK-80I treatment on performance in the radial-arm maze. Animals were given two test runs with a delay of $30 \mathrm{~s}(30 \mathrm{~s})$ or $15 \mathrm{~min}(15 \mathrm{~m}$ ) between them on each trial. (a) The asterisk indicates that animals pretreated with saline and treated with MK-80 I made significantly more revisits to previously baited arms during the first test run relative to animals pretreated and treated with saline and animals pretreated with clonidine $(0.05 \mathrm{mg} / \mathrm{kg})$ and treated with MK-80I $(0.1 \mathrm{mg} / \mathrm{kg})$. (b) Animals made significantly more incorrect choices during the first four arm entries after a I5-min delay relative to a $30 \mathrm{~s}$ delay. (c) After the delay, animals treated with MK-80 I were more likely to visit arms previously baited during the first test run. Animals were also more likely to make such errors after a 15-min delay relative to a 30-s delay. (d) Animals treated with MK-80I revisited more baited arms during the second test run after the delay than did animals treated with saline. The single asterisk (*) indicates significant differences between animals treated with saline relative to animals treated with MK-80I within the same pretreatment condition and same delay interval (ie the bar to the left of the asterisk). Animals pretreated and treated with saline made no revisits after the I5-min delay. Data represent the average number for each measure ( \pm SEM) for each group; $n=6$ per group. 
15-min delay, those treated with saline had fewer arm revisits than those treated with MK-801 (Fishers PLSD, $p<0.05)$. A similar comparison of clonidine-pretreated animals at the 30-s delay revealed that those treated with saline made fewer revisits in comparison to those treated with MK-801 (Fishers PLSD, $p<0.05$ ).

\section{Clonidine Does Not Alleviate the Effects of Hippocampal Damage in the Discrete-Trial Delayed Alternation Task}

In the study of rats with hippocampal damage, it was found that animals in the hippocampal damage group had significantly less hippocampal area than did rats in the sham surgery control group (lesion effect: $\mathrm{F}(1,22)=59.8$, $p<0.0001$ ) (Table 3). Qualitative assessment of the hippocampal damage caused by stereotaxic NMDA infusion indicated that damage was primarily confined to the dorsal hippocampus. Tissue loss was uniform throughout all cell fields within the dorsal hippocampus and most conspicuous in the anterior regions of the dorsal hippocampus (lesion $\times$ location interaction: $\mathrm{F}(3,66)=2.7, \quad p<0.05$ ) (Table 3). Remaining tissue in these areas was marked by gliosis and fewer neurons in comparison to sham controls (Figure 4). The ventral hippocampus in animals with hippocampal damage appeared to be relatively intact.

Approximately 1 month after surgery, animals were trained and tested in the delayed alternation task in a manner identical to that used in the first two experiments. There was an overall delay effect on choice accuracy with animals making more correct choices after the 10-s delay relative to the 40 -s delay (delay effect: $\mathrm{F}(1,48)=1.4$, $p<0.0005$ ) (Figure 5). Hippocampal damage was found to significantly disrupt choice accuracy at both delays (lesion effect: $F(1,29)=7.0$ and $11.2, p<0.01$ and $.002,10$ - and 40 -s delays, respectively). There were no further significant main effects or interactions at the 10-s delay. However, at the 40-s delay, there was a significant effect of clonidine (treatment effect: $F(2,58)=3.1, p=0.05)$ and a treatment $\times$ group interaction (interaction effect: $\mathrm{F}(2,58)=3.4, p<0.04$ ). Rats with hippocampal lesions were found to perform worse than sham rats after treatment with saline (Fishers PLSD, $p<0.02$ ) and the low dose of clonidine (Fishers PLSD, $p<0.0004)$, but not after treatment with the high dose of clonidine (Fishers PLSD, $p=0.14$ ). Post hoc comparisons of choice accuracy in the sham animals treated with saline and the two clonidine doses indicated that sham animals treated with $0.01 \mathrm{mg} / \mathrm{kg}$ of clonidine performed significantly better than animals treated with saline (Fishers PLSD, $p<0.03$ ) or $0.05 \mathrm{mg} / \mathrm{kg}$ of clonidine (Fishers PLSD, $p<0.004$ ). There were no such effects of clonidine on choice accuracy in the animals with hippocampal damage.

\section{DISCUSSION}

The purpose of the studies reported here was to determine if clonidine could alleviate spatial memory deficits produced by the NMDA receptor antagonist, MK-801, and hippocampal damage. These two models of memory loss share some physiological qualities - loss of glutamate function within the hippocampus - but possess obvious differences as well. Despite these differences, they both cause deficits in
Table 3 Hippocampal Area Measurements in Animals with and without Hippocampal Damage

\begin{tabular}{lllll}
\hline & \multicolumn{4}{c}{ Section location posterior from bregma } \\
\cline { 2 - 5 } Group & $\mathbf{- 3 . 1 4} \mathbf{~ m m}$ & $\mathbf{- 3 . 6 0} \mathbf{~ m m}$ & $\mathbf{- 4 . 3 0} \mathbf{~ m m}$ & $\mathbf{- 5 . 2 0} \mathbf{~ m m}$ \\
\hline Sham (I3) & $4.7 \pm 0.2$ & $5.5 \pm 0.2$ & $6.5 \pm 0.3$ & $17.8 \pm 0.4$ \\
Lesion (I I) & $1.6 \pm 0.3 *$ & $2.2 \pm 0.4 *$ & $3.5 \pm 0.6 *$ & $16.5 \pm 0.8$ \\
\hline
\end{tabular}

The lesion group had significantly less hippocampal area than the sham control group (as indicated by the asterisks), except in the most posterior section.

Sections were measured in the coronal plane. Coordinates (from bregma) are taken from Paxinos and Watson (1986). Area measurements are presented in $\mathrm{mm}^{2} \pm$ SEM. Number per group indicated in parentheses.

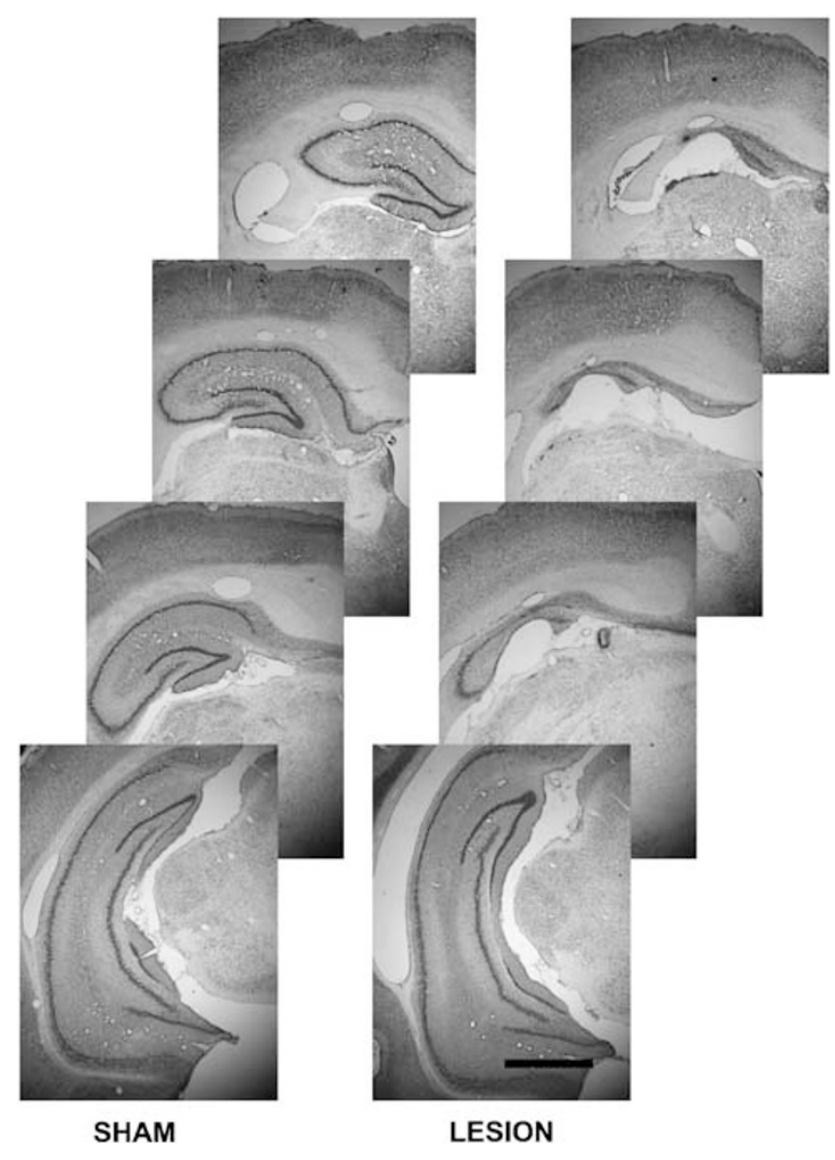

Figure 4 Photomicrographs of the hippocampus from a sham control animal and an animal with a hippocampal lesion at four anterior-posterior locations in the brain. Note that tissue loss is mainly confined to the dorsal hippocampus in animals with hippocampal damage. Scale bar $=1 \mathrm{~mm}$.

spatial working memory. The data demonstrate that clonidine effectively ameliorated the memory deficits produced by MK- 801 in two tests of spatial memory, the discrete-trial delayed spatial alternation task and the radialarm maze. These results replicate and extend earlier work showing the cognitive-enhancing effects of clonidine in animals treated with PCP (Jentsch and Anzivino, 2004; Marrs et al, 2005; McCann et al, 1987). However, clonidine did not improve memory in rats with hippocampal damage. These findings suggest that clonidine is effective in treating memory impairment, but in a pathology-specific manner. 

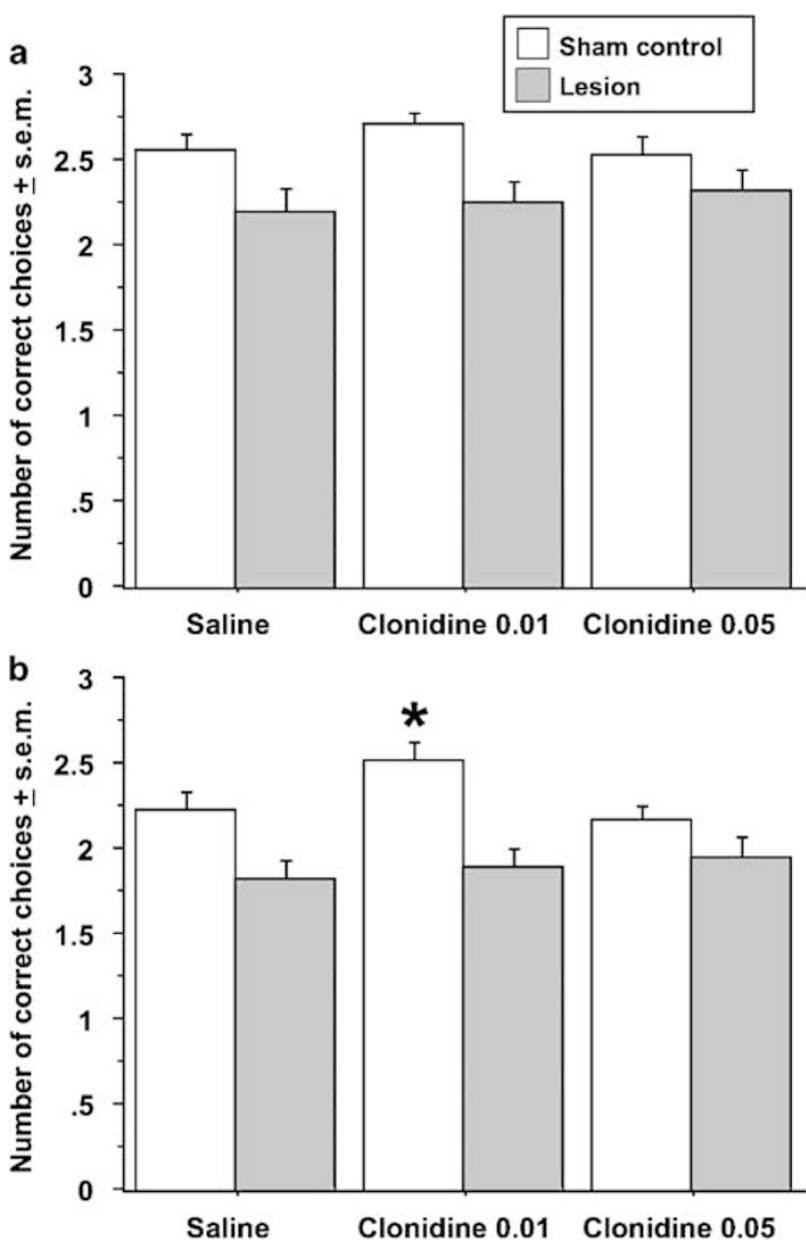

Figure 5 Effects of clonidine treatment on deficits produced by hippocampal damage in the discrete-trial delayed alternation task. Data from the 10-s delay interval are represented in (a), and data from the 40-s interval are represented in (b). Animals with hippocampal damage made significantly fewer correct choices than sham controls at each drug dose and delay interval tested except for the comparison of the two groups at the 40 -s delay after treatment with $0.05 \mathrm{mg} / \mathrm{kg}$ of clonidine. At the $40-\mathrm{s}$ interval, sham animals treated with the low dose of clonidine made significantly more correct choices (as indicated by the asterisk) than did sham animals treated with saline or the high dose of clonidine. Data represent the average number of correct choices ( \pm SEM) for each group at each delay out of three trials; $n=13$ in the hippocampal damage and $n=16$ in the sham control group.

Apparently, clonidine is capable of counteracting memory impairment associated with states of broad-scale glutamate hypofunction but is incapable of correcting memory impairment related to a dramatic reduction in hippocampal volume.

Several previous reports have indicated that clonidine and the related $\alpha_{2}$ agonist, guanfacine, can reverse spatial memory impairments and other forms of memory deficits produced by PCP treatment (Jentsch and Anzivino, 2004; Marrs et al, 2005; McCann et al, 1987). While impressive, these findings nonetheless raise questions about whether the cognitive-enhancing actions of $\alpha_{2}$ agonists would be observed after treatment with other NMDA antagonists, such as MK-801. While PCP and MK-801 serve as noncompetitive antagonists of the NMDA receptor, their physiological and behavioral effects are known to be somewhat different (Jacobs et al, 2000; Ogren and Goldstein, 1994). Furthermore, the ability of clonidine to block the amnestic effects of PCP has only been shown in operant paradigms (Jentsch and Anzivino, 2004; Marrs et al, 2005) and in the radial-arm maze (McCann et al, 1987). The results of the present study suggest that the pro-cognitive effects of clonidine extend to other NMDA antagonists, such as MK-801, and can be observed in other paradigms, such as delayed spatial alternation.

MK-801 is well known to increase locomotor activity and produce measurable sensorimotor disturbances in rats at the dose used in the present study (eg Jacobs et al, 2000). These effects make it plausible that working memory may remain functional in MK-801-treated rats, but is masked by disruptions in basic sensorimotor processes. Three observations suggest this explanation may only partially account for deficits in the delayed alternation task. First, the time to choose in the delayed alternation task in first two experiments was not significantly altered by MK-801 treatment, indicating that basic processes such as initiation of movement and ambulatory movement were not dramatically affected in the T-maze task by MK-801. Second, if sensorimotor disturbances produced by MK-801 adversely affect performance in the T-maze, one could expect to find a significant correlation between the time to choose and choice accuracy. Such correlations between the time to choose and choice accuracy in the high dose MK-801treated rats pretreated with saline were not found to be significant (first experiment: $r=-0.20, p=0.47$; second experiment: $\mathrm{r}=-0.46, p=0.13$ ). One could argue that the time to choose measure is not a very sensitive measure of sensorimotor disturbances; however, this measure is sensitive to the effects of other treatments known to alter sensorimotor function such as antipsychotic drugs (Bardgett et al, 2006a,b). Finally, the pattern of effects exerted by clonidine and MK-801 upon the time to choose are not consistent with their effects on choice accuracy, suggesting that the former does not account for the latter.

The mechanism underlying clonidine's positive effect on memory in MK-801-treated rats cannot be ascertained by our behavioral results. Clonidine has affinity for each $\alpha_{2}$ receptor agonist subtype. It is not clear which receptor subtype accounts for clonidine's ability to alleviate the effects of MK-801 on memory, although previous studies in mice and monkeys have suggested the $\alpha_{2 \mathrm{~A}}$ or $\alpha_{2 \mathrm{C}}$ subtypes as likely mechanisms for cognitive enhancement (Franowicz and Arnsten, 2002; Franowicz et al, 2002). In terms of the anatomical locus of clonidine's action in the present studies, it is probable that clonidine suppresses the disruptive effects of MK-801 on prefrontal cortical function. Jackson et al (2004) have shown that MK-801, at the doses used in the present study, increases irregular firing and decreases organized burst firing of neurons in the prefrontal cortex. These chaotic changes in neuronal firing may reflect excessive cortical neurotransmitter (eg acetylcholine, glutamate, dopamine) release evoked by NMDA antagonists (Kim et al, 1999; Moghaddam and Adams, 1998) Clonidine, on the other hand, has been shown to suppress elevations in neurotransmitter release induced by various perturbations, including MK-801(Acquas et al, 1998; Jellish et al, 2005; Kim et al, 1999). Clonidine's suppressive effect on neurotransmitter release may be due to its direct action 
on presynaptic terminals (Kamisaki et al, 1992) or indirect effects on neuronal firing (Kawahara et al, 2001). Alternatively, clonidine may act at post-synaptic $\alpha_{2}$ receptors, since the loss of noradrenergic terminals actually enhances the pro-cognitive effects of clonidine (see Arnsten (2004) for review). Nonetheless, when taken together, the previous studies appear to suggest that clonidine reduces cortical noise generated by NMDA antagonists and enhances neuronal signals related to normal memory function. In support of this hypothesis, clonidine has been shown at a neuronal level to strengthen firing in the prefrontal cortex during delay-response periods and, at a behavioral level, to decrease distractibility during memory testing (see Arnsten (2004) for review).

While clonidine had very clear effects on delayed alternation deficits induced by MK-801, its impact upon impairments caused by MK-801 in the radial-arm maze task was less dramatic. Clonidine was found to prevent MK-801treated rats from making more arm revisits during the first run of each test trial. However, while there were significant effects of MK-801 on the number of retroactive errors and arm revisits during the second run of each trial, clonidine was unable to alleviate these effects. There are several ways to tie these results together. First, it is possible that the radial-arm maze data was underpowered and limited in its ability to yield significant group differences. However, the data from these animals reflect a fairly large number of trials (ie the average of four trials performed under each pretreatment-treatment condition for each delay). Moreover, the significant interaction between clonidine and MK-801 for the revisit data from the first run suggests that the experiment had adequate power. The second interpretation is that the different behavioral measures used in the radialarm maze testing reflect different forms of memory or amounts of memory burden. MK-801 did not alter the number of correct choices within the first four choices during the second run of each trial, suggesting that while this form of choice behavior is sensitive to delays, it is either insensitive to NMDA receptor blockade or difficult for even control animals. The other measures used in the second run (retroactive errors and arm revisits) were sensitive to MK801 treatment yet involved a larger number of choices and the possibility of proactive interference from the first run in contrast to the arm revisits during the first run. This latter interpretation may provide some parsimony for the positive effects of clonidine on first run revisits in the radial-arm maze and on delayed alternation, since both tasks involve limited choices and relatively little proactive interference.

While NMDA hypofunction may serve as one pathophysiological mechanism that accounts for poor memory performance in several neuropsychiatric disorders (Lewis and Moghaddam, 2006; Olney and Farber, 1995; Olney et al, 1998), reduced hippocampal volume represents yet another mechanism that could contribute to performance deficits in these disorders (Grundman et al, 2002; Gur et al, 2000; Heckers, 2001; Kohler et al, 1998). Attempts to emulate disorder-related reductions of hippocampal volume in animals have been hampered in part by limited agreement on the exact nature and scale of the topographical, neurochemical, and functional alterations found within the hippocampus of people with such disorders. Along the same lines, most animal models of human hippocampal deficits involve rather broad-scale, indiscriminant destruction of hippocampal tissue in comparison to the more limited volume reductions reported in the human literature. Nonetheless, it would seem that if treatments can alleviate significant behavioral impairment in animals with hippocampal damage, such treatments may have utility for similar behavioral deficits in people with more circumscribed reductions in hippocampal volume. With this idea in mind, studies from our own laboratory have demonstrated that excitotoxic damage to the hippocampus results in significant hippocampal tissue loss and profound spatial working memory deficits (Bardgett et al, 2006a, b).

In the present study, animals with excitotoxic damage to the hippocampus experienced dramatic tissue loss in the anterior dorsal hippocampus. While the neurochemical status of the remaining tissue was not addressed in this study, previous work has shown that excitotoxic hippocampal damage is accompanied by subtle losses in microtubule-associated protein-2 and more significant decreases in staining for the GluR1 and GluR2/3 glutamate receptor subunits (Csernansky et al, 1998). A recent review has provided strong evidence for a pivotal role for the GluR2 subunit in synaptic plasticity (Isaac et al, 2007), suggesting that decreases in this receptor subunit may be important in the memory loss observed in rats with excitotoxic damage. The loss of excitatory hippocampal neurons creates a hypoglutamatergic state, analogous to the effects of MK-801 on hippocampal function, but excitotoxic damage to hippocampus obviously disrupts all neurochemical processes within the hippocampus as well as the circuitry in which the hippocampus participates.

To date, it is unknown whether the memory impairment produced by hippocampal damage is sensitive to the procognitive actions of drugs, such as clonidine, that seem to alleviate the untoward effects of NMDA hypofunction on memory. Earlier work by Ammassari-Teule et al (1991) found that clonidine could reverse spatial learning deficits in rats with partial fornix lesions. Such damage presumably alters the neurochemical composition of the hippocampus due to the disruption of subcortical afferent pathways that supply neurotransmitters to the hippocampus. However, the mechanisms driving memory impairment in rats with partial fornix transection are likely to be qualitatively different from those responsible for memory problems in rats with direct damage to the hippocampus, and may explain why rats with hippocampal damage failed to respond to clonidine in the present study.

The implications of the data from rats with hippocampal damage are twofold. First, they may indirectly suggest which brain regions play a role in clonidine's memoryimproving effects. Given that rats with hippocampal damage did not benefit from clonidine, one could speculate that perhaps part of clonidine's cognitive-enhancing ability involves its direct effects on the hippocampus, such as reducing norepinephrine release (Sacchetti et al, 2001), decreasing excitatory neurotransmission (Boehm, 1999), or altering $\theta$ activity (Ammassari-Teule et al, 1991). However, this interpretation merits caution. While Ammassari-Teule et al (1991) found that clonidine alleviates radial-arm maze deficits in rats with fornix transactions, other studies (Arnsten and Goldman-Rakic, 1990; Sirvio et al, 1991) have shown that clonidine and guanfacine do not improve 
performance in tasks, such as delayed non-matching to sample and the swim maze, that rely on intact hippocampal function. These studies would seem to suggest that clonidine does not act upon the hippocampus to improve memory. Regardless of where clonidine acts within the brain to improve spatial working memory in rats treated with MK-801, these effects are apparently not sufficient to counteract the negative consequences of reduced hippocampal volume on memory. It should be noted that clonidine's ineffectiveness in the hippocampal model is not due to an inherent inability of any treatment to improve memory in rats with hippocampal damage. Recent studies from our laboratory have shown that the atypical antipsychotics, clozapine and risperidone, can improve spatial working memory in rats with hippocampal damage (Bardgett et al, 2006a, b).

It would be interesting to determine if memory loss produced by smaller scale damage to the hippocampus would be more sensitive to the positive effects of clonidine on memory, although the trade-off with such an approach may be less reliable memory deficits. It would also be interesting to determine if memory loss produced by cellfield specific damage within the hippocampus is sensitive to clonidine in a site-specific manner. Finally, as discussed above, it is possible that the clonidine's ability to improve memory in rats treated with MK-801 requires the hippocampus as a substrate; however, testing such a hypothesis by assessing the effects of hippocampal lesions on MK-801treated rats would involve confounds with lesion-induced memory deficits. Indirectly related to this latter idea, the inability of clonidine to improve memory in the hippocampal model relative to the MK-801 model is noteworthy since the magnitude of the memory deficits observed in the former model was less than that observed in the latter. It is tempting to speculate that the greater deficit observed in the MK-801 model is simply due to a more distributed disruption of neurotransmission throughout the brain $v s$ the discrete disruption of neurotransmission within the hippocampus produced in rats with hippocampal damage. Nonetheless, the performance decrement observed in the latter model should not be viewed as marginal since it was on a scale similar to that observed in previous studies of discrete-trial delayed alternation in rats with hippocampal damage (Bardgett et al, 2006a,b) and approached chance levels at the 40-s delay interval. It is possible that larger scale volume loss could have triggered greater reductions in performance; however, it was one of our goals to produce a degree of volume loss more consistent with the degree of volume reduction observed in neuropsychiatric disorders and not characterized by the complete destruction of hippocampal tissue. One could argue that the difference between the magnitude of memory loss in the MK-801 and hippocampal models is simply quantitative, which would imply that the two models are similar in mechanism. However, this interpretation makes little sense given the positive effects of clonidine on the more severe deficits observed in the MK- 801 model. The simplest explanation of the present results is that MK- 801 and hippocampal damage can produce significant deficits in the same test of memory, but since only the former model is sensitive to clonidine, the memory deficits observed in each model emanate from different pathophysiological processes.
If different forms of pathophysiology lead to the same memory deficit, then each form may require a distinct form of treatment. This simple conclusion bears noting. As discussed in the Introduction, many animal models of memory deficits linked to neuropsychiatric disorders have been developed, and some labs, including our own, have championed one model over another. However, our results suggest that one model may not be necessarily better than the other - instead, they are better together. This sentiment is consistent with recent recommendations for more diverse approaches to modeling cognitive impairment in animals (Floresco et al, 2005; Green et al, 2005). As new pathophysiological pathways are identified and new animal models developed, treatments for these distinct pathophysiologies can be identified and the symptoms of more individuals with memory impairment effectively managed.

\section{ACKNOWLEDGEMENTS}

Support from the National Center for Research Resources Grant P20 RR16481 and the National Institute of Mental Health Grant R15 MH076788 is gratefully acknowledged. We would like to thank John Csernansky for his reading of the manuscript and helpful suggestions.

\section{DISCLOSURE/CONFLICT OF INTEREST}

The authors declare that this research has been funded by the NIH-National Center for Research Resources and the National Institute of Mental Health. The authors declare that Dr Bardgett has performed contractual preclinical research for Janssen Pharmaceutical. All other authors declare that, except for income received from primary employers, no financial support or compensation has been received from any individual or corporate entity over the past 3 years for research or professional service and there are no personal financial holdings that could be perceived as constituting a potential conflict of interest.

\section{REFERENCES}

Acquas E, Wilson C, Fibiger HC (1998). Pharmacology of sensory stimulation-evoked increases in frontal cortical acetylcholine release. Neuroscience 85: 73-83.

Adams B, Moghaddam B (1998). Corticolimbic dopamine neurotransmission is temporally dissociated from the cognitive and locomotor effects of phencyclidine. J Neurosci 18: 5545-5554.

Ammassari-Teule M, Maho C, Sara SJ (1991). Clonidine reverses spatial learning deficits and reinstates theta frequencies in rats with partial fornix section. Behav Brain Res 45: 1-8.

Arnsten AF (2004). Adrenergic targets for the treatment of cognitive deficits in schizophrenia. Psychopharmacology 174: 25-31.

Arnsten AF, Goldman-Rakic PS (1985). Alpha 2-adrenergic mechanisms in prefrontal cortex associated with cognitive decline in aged nonhuman primates. Science 230: 1273-1276.

Arnsten AF, Goldman-Rakic PS (1990). Analysis of alpha-2 adrenergic agonist effects on the delayed nonmatch-to-sample performance of aged rhesus monkeys. Neurobiol Aging 11: 583-590.

Aultman JM, Moghaddam B (2001). Distinct contributions of glutamate and dopamine receptors to temporal aspects of rodent 
working memory using a clinically relevant task. Psychopharmacology 153: 353-364.

Aura J, Riekkinen Jr P (1999). Blockade of NMDA receptors located at the dorsomedial prefrontal cortex impairs spatial working memory in rats. Neuroreport 10: 243-248.

Bardgett ME, Baum KT, O'Connell SM, Lee NM, Hon JC (2006a). Effects of risperidone on locomotor activity and spatial memory in rats with hippocampal damage. Neuropharmacology 51: 1156-1162.

Bardgett ME, Boeckman R, Krochmal D, Fernando H, Ahrens R, Csernansky JG (2003). NMDA receptor blockade and hippocampal neuronal loss impair fear conditioning and position habit reversal in C57Bl/6 mice. Brain Res Bull 60: 131-142.

Bardgett ME, Griffith MS, Foltz RF, Hopkins JA, Massie CM, O'Connell SM (2006b). The effects of clozapine on delayed spatial alternation deficits in rats with hippocampal damage. Neurobiol Learn Mem 85: 86-94.

Bernaerts P, Lamberty Y, Tirelli E (2004). Histamine H3 antagonist thioperamide dose-dependently enhances memory consolidation and reverses amnesia induced by dizocilpine or scopolamine in a one-trial inhibitory avoidance task in mice. Behav Brain Res 154: 211-219.

Boehm S (1999). Presynaptic alpha2-adrenoceptors control excitatory, but not inhibitory, transmission at rat hippocampal synapses. J Physiol 519: 439-449.

Csernansky JG, Csernansky CA, Kogelman L, Montgomery EM, Bardgett ME (1998). Progressive neurodegeneration after intracerebroventricular kainic acid administration in rats: implications for schizophrenia? Biol Psychiatry 44: 1143-1150.

Csernansky JG, Martin M, Shah R, Bertchume A, Colvin J, Dong H (2005). Cholinesterase inhibitors ameliorate behavioral deficits induced by MK-801 in mice. Neuropsychopharmacology 30: 2135-2143.

Csernansky JG, Wang L, Joshi S, Miller JP, Gado M, Kido D et al (2000). Early DAT is distinguished from aging by highdimensional mapping of the hippocampus. Dementia of the Alzheimer type. Neurology 55: 1636-1643.

Dudchenko PA (2001). How do animals actually solve the T maze? Behav Neurosci 115: 850-860.

Floresco SB, Geyer MA, Gold LH, Grace AA (2005). Developing predictive animal models and establishing a preclinical trials network for assessing treatment effects on cognition in schizophrenia. Schizophr Bull 31: 888-894.

Franowicz JS, Arnsten AF (2002). Actions of alpha-2 noradrenergic agonists on spatial working memory and blood pressure in rhesus monkeys appear to be mediated by the same receptor subtype. Psychopharmacology 162: 304-312.

Franowicz JS, Kessler LE, Borja CM, Kobilka BK, Limbird LE, Arnsten AF (2002). Mutation of the alpha2A-adrenoceptor impairs working memory performance and annuls cognitive enhancement by guanfacine. J Neurosci 22: 8771-8777.

Friedman JI, Adler DN, Temporini HD, Kemether E, Harvey PD, White L et al (2001). Guanfacine treatment of cognitive impairment in schizophrenia. Neuropsychopharmacology 25: 402-409.

Green MF, Olivier B, Crawley JN, Penn DL, Silverstein S (2005). Social cognition in schizophrenia: recommendations from the measurement and treatment research to improve cognition in schizophrenia new approaches conference. Schizophr Bull 31: 882-887.

Grundman M, Sencakova D, Jack Jr CR, Petersen RC, Kim HT, Schultz A et al (2002). Brain MRI hippocampal volume and prediction of clinical status in a mild cognitive impairment trial. J Mol Neurosci 19: 23-27.

Gur RE, Turetsky BI, Cowell PE, Finkelman C, Maany V, Grossman RI et al (2000). Temporolimbic volume reductions in schizophrenia. Arch Gen Psychiatry 57: 769-775.
Heckers S (2001). Neuroimaging studies of the hippocampus in schizophrenia. Hippocampus 11: 520-528.

Hyman SE, Fenton WS (2003). What are the right targets for psychopharmacology? Science 299: 350-351.

Isaac JT, Ashby M, McBain CJ (2007). The role of the GluR2 subunit in AMPA receptor function and synaptic plasticity. Neuron 54: 859-871.

Jackson ME, Homayoun H, Moghaddam B (2004). NMDA receptor hypofunction produces concomitant firing rate potentiation and burst activity reduction in the prefrontal cortex. Proc Natl Acad Sci 101: 8467-8472.

Jacobs PS, Taylor BM, Bardgett ME (2000). Maturation of locomotor and Fos responses to the NMDA antagonists, PCP and MK-801. Dev Brain Res 122: 91-95.

Jellish WS, Murdoch J, Kindel G, Zhang X, White FA (2005). The effect of clonidine on cell survival, glutamate, and aspartate release in normo- and hyperglycemic rats after near complete forebrain ischemia. Exp Brain Res 167: 526-534.

Jentsch JD, Anzivino LA (2004). A low dose of the alpha2 agonist clonidine ameliorates the visual attention and spatial working memory deficits produced by phencyclidine administration to rats. Psychopharmacology 175: 76-83.

Kamisaki Y, Hamahashi T, Hamada T, Maeda K, Itoh T (1992). Presynaptic inhibition by clonidine of neurotransmitter amino acid release in various brain regions. Eur J Pharmacol 217: 57-63.

Kawahara H, Kawahara Y, Westerink BH (2001). The noradrenaline-dopamine interaction in the rat medial prefrontal cortex studied by multi-probe microdialysis. Eur J Pharmacol 418: 177-186.

Keseberg U, Schmidt WJ (1993). Tetrahydroaminoacridine attenuates dizocilpine-induced behavioural changes. Behav Brain Res 53: 113-118.

Kesner RP, Rogers J (2004). An analysis of independence and interactions of brain substrates that subserve multiple attributes, memory systems, and underlying processes. Neurobiol Learn Mem 82: 199-215.

Kim SH, Price MT, Olney JW, Farber NB (1999). Excessive cerebrocortical release of acetylcholine induced by NMDA antagonists is reduced by GABAergic and alpha2-adrenergic agonists. Mol Psychiatry 4: 344-352.

Kohler S, Black SE, Sinden M, Szekely C, Kidron D, Parker JL et al (1998). Memory impairments associated with hippocampal versus parahippocampal-gyrus atrophy: an MR volumetry study in Alzheimer's disease. Neuropsychologia 36: 901-914.

Kretschmer BD, Fink S (1999). Spatial learning deficit after NMDA receptor blockade and state-dependency. Behav Pharmacol 10: 423-428.

Lewis DA, Moghaddam B (2006). Cognitive dysfunction in schizophrenia: convergence of gamma-aminobutyric acid and glutamate alterations. Arch Neurol 63: 1372-1376.

Li BM, Mao ZM, Wang M, Mei ZT (1999). Alpha-2 adrenergic modulation of prefrontal cortical neuronal activity related to spatial working memory in monkeys. Neuropsychopharmacology 21: 601-610.

Liu P, Bilkey DK (1999). The effect of excitotoxic lesions centered on the perirhinal cortex in two versions of the radial arm maze task. Behav Neurosci 113: 672-682.

Marcus MM, Jardemark KE, Wadenberg ML, Langlois X, Hertel P, Svensson TH (2005). Combined alpha2 and D2/3 receptor blockade enhances cortical glutamatergic transmission and reverses cognitive impairment in the rat. Int J Neuropsychopharmacol 8: 315-327.

Marrs W, Kuperman J, Avedian T, Roth RH, Jentsch JD (2005). Alpha-2 adrenoceptor activation inhibits phencyclidine-induced deficits of spatial working memory in rats. Neuropsychopharmacology 30: 1500-1510. 
McCann DJ, Rabin RA, Winter JC (1987). Interactions of clonidine with phencyclidine and ketamine: studies of radial maze performance and righting reflex in rats. Pharmacol Biochem Behav 26: 23-28.

McCarley RW, Wible CG, Frumin M, Hirayasu Y, Levitt JJ, Fischer IA et al (1999). MRI anatomy of schizophrenia. Biol Psychiatry 45: 1099-1119.

Moghaddam B, Adams B, Verma A, Daly D (1997). Activation of glutamatergic neurotransmission by ketamine: a novel step in the pathway from NMDA receptor blockade to dopaminergic and cognitive disruptions associated with the prefrontal cortex. J Neurosci 17: 2921-2927.

Moghaddam B, Adams BW (1998). Reversal of phencyclidine effects by a group II metabotropic glutamate receptor agonist in rats. Science 281: 1349-1352.

National Research Council (1996). Guide for the Care and Use of Laboratory Animals. National Academy Press: Washington DC.

Newcomer JW, Farber NB, Jevtovic-Todorovic V, Selke G, Melson AK, Hershey $\mathrm{T}$ et al (1999). Ketamine-induced NMDA receptor hypofunction as a model of memory impairment and psychosis. Neuropsychopharmacology 20: 106-118.

O’Donnell P, Lewis BL, Weinberger DR, Lipska BK (2002). Neonatal hippocampal damage alters electrophysiological properties of prefrontal cortical neurons in adult rats. Cereb Cortex 12: $975-982$

Ogren SO, Goldstein M (1994). Phencyclidine- and dizocilpineinduced hyperlocomotion are differentially mediated. Neuropsychopharmacology 11: 167-177.

Olney JW, Farber NB (1995). Glutamate receptor dysfunction and schizophrenia. Arch Gen Psychiatry 52: 998-1007.

Olney JW, Wozniak DF, Farber NB (1998). Glutamate receptor dysfunction and Alzheimer's disease. Restor Neurol Neurosci 13: 75-83.

Paxinos G, Watson C (1986). The Rat Brain in Stereotaxic Coordinates, 2nd edn. Academic Press: Sydney.
Riekkinen M, Laakso MP, Jakala P (1999). Clonidine impairs sustained attention and memory in Alzheimer's disease. Neuroscience 92: 975-982.

Riekkinen Jr P, Riekkinen M (1999). THA improves word priming and clonidine enhances fluency and working memory in Alzheimer's disease. Neuropsychopharmacology 20: 357-364.

Roe DL, Bardgett ME, Csernansky CA, Csernansky JG (1998). Induction of fos protein by antipsychotic drugs in rat brain following kainic acid-induced limbic-cortical neuronal loss. Psychopharmacology 138: 151-158.

Sacchetti G, Bernini M, Gobbi M, Parini S, Pirona L, Mennini T et al (2001). Chronic treatment with desipramine facilitates its effect on extracellular noradrenaline in the rat hippocampus: studies on the role of presynaptic alpha2-adrenoceptors. Naunyn Schmiedebergs Arch Pharmacol 363: 66-72.

Sheline YI, Wang PW, Gado MH, Csernansky JG, Vannier MW (1996). Hippocampal atrophy in recurrent major depression. Proc Natl Acad Sci 93: 3908-3913.

Sirvio J, Riekkinen Jr P, Vajanto I, Koivisto E, Riekkinen PJ (1991). The effects of guanfacine, alpha-2 agonist, on the performance of young and aged rats in spatial navigation task. Behav Neural Biol 56: 101-107.

Wang M, Ramos BP, Paspalas CD, Shu Y, Simen A, Duque A et al (2007). Alpha2A-adrenoceptors strengthen working memory networks by inhibiting cAMP-HCN channel signaling in prefrontal cortex. Cell 129: 397-410.

Yoshihara T, Ichitani Y (2004). Hippocampal N-methyl-Daspartate receptor-mediated encoding and retrieval processes in spatial working memory: delay-interposed radial maze performance in rats. Neuroscience 129: 1-10.

Zhang HT, Huang Y, Suvarna NU, Deng C, Crissman AM, Hopper AT et al (2005). Effects of the novel PDE4 inhibitors MEM1018 and MEM1091 on memory in the radial-arm maze and inhibitory avoidance tests in rats. Psychopharmacology 179: 613-619. 